\title{
ON THE EXISTENCE OF GLOBAL TCHEBYCHEV NETS
}

\author{
SANDRA L. SAMELSON AND W. P. DAYAWANSA
}

ABstract. Let $S$ be a complete, open simply connected surface. Suppose that the integral of the Gauss curvature over arbitrary measurable sets is less than $\pi / 2$ in magnitude. We show that the surface admits a global Tchebychev net.

\section{INTRODUCTION}

The mathematical analysis of the deformation of certain types of reinforced networks has recently attracted attention. In one such network an initially planar, orthogonal set of fibers is deformed into a curved network in space. For simplicity of analysis it is generally assumed that the network consists of a continuum of fibers, thus forming a surface. Such a surface is called a Tchebychev net [Pip81].

From a mathematical point of view a (global) Tchebychev net can be described as a system of local coordinates on a two-dimensional surface in $\Re^{3}$ such that the coordinate vector fields have unit magnitudes and the coordinate transformations are mere translations. Alternatively one can describe it as a commuting frame field of unit magnitude.

Tchebychev [Tch78] introduced the concept of Tchebychev nets in order to model the deformation of cloth. More recently, Tchebychev nets have been used to model curved surfaces of structures with fiber reinforcements [Riv59], [Pip81], etc. One of the possible modern applications of Tchebychev nets is in the construction of surfaces from graphite-epoxy composite materials. This type of material is constructed by "weaving" layers of carbon fibers in order to produce material elements of desirable shapes.

Here we focus on the global aspects of the problem. We assume that our surface is complete, open and simply connected (hence diffeomorphic to $\Re^{2}$ ), and we ask whether or not we can put a global coordinate chart such that the coordinate vector fields are of unit length.

A formula due to Hazzidakis [Haz80] asserts that on a Techebychev net the integral of the Gauss curvature over an arbitrary coordinate rectangle should be

Received by the editors May 4, 1992 and, in revised form, August 25, 1993; originally communicated to the Proceedings of the AMS by Christopher Croke.

1991 Mathematics Subject Classification. Primary 58G16, 58G30; Secondary 53C21.

Key words and phrases. Tchebychev nets, curvature bounds.

The first author was partially supported by the United States Army under contract number: DAAL03-89-C-0038 to the University of Minnesota.

The second author was partially supported by the National Science Foundation Engineering Research Center Program Grant NSFD 8803012, and the NSF Grant ECS 9096121. 
less than $2 \pi$ in magnitude. Therefore, the answer to our question is no in general. This confirms the intutive notion that in order to fit a cloth over a surface, its Gaussian curvature should not be too high. The main result contained in this paper is that if the integral of the Gauss curvature of arbitrary measurable subsets of the surface is less than $\pi / 2$ in magnitude, then the surface admits a global Tchebychev net.

\section{Notation}

We consider a complete open simply connected $C^{\infty}$ surface $S$. Since $S$ is diffeomorphic to $\Re^{2}$, without loss of generality we take $S=\Re^{2}$ and let $\left\{\left(u_{1}, u_{2}\right)\right\}_{\left(u_{1}, u_{2}\right) \in \Re^{2}}$ be any convenient coordinate system on $S$. Let $g=$ $\sum_{1 \leq i, j \leq 2} g_{i j} d u^{i} d u^{j}$ be the Riemannian metric tensor on $\Re^{2}$ (so $g_{i j}$ are realvalued $C^{\infty}$ functions on $\Re^{2}$ ).

$\nabla:$ Levi-Civita connection,

$\left\{\Gamma_{i j}^{k}\right\}_{1 \leq i, j, k \leq 2}:$ Christoffel symbols,

$\kappa$ : Gauss curvature,

$d A$ : Riemannian Area element $\left(=\sqrt{g_{11} g_{22}-g_{12}^{2}} d u^{1} d u^{2}\right)$,

$S^{+}=\left\{x \in \Re^{2}: \kappa(x) \geq 0\right\}$,

$S^{-}=\left\{x \in \Re^{2}: \kappa(x) \leq 0\right\}$.

\section{Preliminaries}

Definition 3.1. A Tchebychev net or $\left(\Re^{2}, g\right)$ is a global coordinate chart $\left\{\Re^{2}\right.$, $\left.\left(x^{1}, x^{2}\right)\right\}$ such that $\left\|\frac{\partial}{\partial x^{i}}\right\|_{g}=1, i=1,2$.

The problem posed here is to decide whether or not it is possible to find a global coordinate system $\left\{\left(x_{1}, x_{2}\right)\right\}_{\left(x_{1}, x_{2}\right) \in \Re^{2}}$ which is a Tchebychev net on $\left(\Re^{2}, g\right)$.

It follows easily from Definition 3.1 that $\nabla_{\frac{\partial}{\partial x^{i}}} \frac{\partial}{\partial x^{j}}=0$ if $i \neq j$, where $\nabla$ is the Levi-Civita connection associated to $g$, i.e., $\frac{\partial}{\partial x^{1}}$ is parallel along $\frac{\partial}{\partial x^{2}}$ and vice-versa.

Let $u=\left(u^{1}, u^{2}\right)$ be any given convenient coordinate system of $\Re^{2}$. The Riemannian metric and Christoffel symbols are given as in section 2 (in the given $\left\{u_{1}, u_{2}\right\}$ coordinate system). Let $\left\{x_{1}, x_{2}\right\}$ denote an unknown coordinate system which gives a Tchebychev net on $\left(\Re^{2}, g\right)$. Let us consider $\left(u_{1}, u_{2}\right)$ as unknown functions of $\left(x_{1}, x_{2}\right)$. Then, the equations $\nabla_{\frac{\partial}{\partial x^{i}}} \frac{\partial}{\partial x^{j}}=0, i \neq j$, can be simplified to obtain Servants' equation

$$
\frac{\partial u^{i}}{\partial x^{1} \partial x^{2}}+\sum_{k, l=1}^{2} \Gamma_{k, \ell}^{i}(u) \frac{\partial u^{k}}{\partial x^{1}} \frac{\partial u^{\ell}}{\partial x^{2}}=0 ; \quad i=1,2 .
$$

Conversely (3.1) implies that $\nabla_{\frac{\partial}{\partial x^{i}}} \frac{\partial}{\partial x^{j}}=0$ if $i \neq j$. In particular, $\left\|\frac{\partial}{\partial x^{i}}\right\|_{g}$ is constant along the integral curves of $\frac{\partial}{\partial x^{j}}$ for $i \neq j$. For later use let $y^{1}=$ 
$x^{1}+x^{2}$ and $y^{2}=x^{1}-x^{2}$ and rewrite (3.1) as:

$$
\frac{\partial^{2} u^{i}}{\partial\left(y^{1}\right)^{2}}-\frac{\partial^{2} u^{i}}{\partial\left(y^{2}\right)^{2}}+\sum_{k, l=1}^{2} \Gamma_{k, \ell}^{i}(u)\left(\frac{\partial u^{\kappa}}{\partial y^{1}} \frac{\partial u^{\ell}}{\partial y^{1}}-\frac{\partial u^{\kappa}}{\partial y^{2}} \frac{\partial u^{\ell}}{\partial y^{2}}\right)=0
$$

$$
i=1,2 .
$$

\section{MAIN THEOREM}

Theorem 4.1. Suppose that $\left(\Re^{2}, g\right)$ is complete and that $\frac{-\pi}{2}<\int_{S^{-}} \kappa d A$ and $\int_{S^{+}} \kappa d A<\frac{\pi}{2}$. Then $\left(\Re^{2}, g\right)$ admits a Tchebychev net.

In the remainder of the paper we will prove this theorem. We will borrow a construction due to Ladyzhenskaya and Shubov [LS84] to prove the global existence of a solution to Servants' equations. Then we show that under the hypothesis in Theorem 4.1 this solution corresponds to a global Tchebychev net.

\section{Proof of the Main theorem}

5.1. Geodesics of $\left(\Re^{2}, g\right)$. The plan is to prove that equation (3.2) admits a solution such that the line $y^{2}=0$ gets mapped onto a geodesic and such that $\frac{\partial}{\partial y^{2}}$ is orthogonal to this geodesic. This allows us to relate the integral of the Gauss curvature to the angle between $\frac{\partial}{\partial x^{1}}$ and $\frac{\partial}{\partial x^{2}}$.

Throughout this section we assume the following:

$$
\frac{-\pi}{2}<\int_{S^{-}} \kappa d A \leq \int_{S^{+}} \kappa d A<\frac{\pi}{2} .
$$

We first show that a geodesic of $\left(\Re^{2}, g\right)$ is closed as a subset of $\Re^{2}$ and hence separates $\Re^{2}$ into two connected components. Let $\rho$ denote the topological metric induced by $g$.

Lemma 5.1. Suppose that $\sigma: \Re \rightarrow \Re^{2}$ is a geodesic. Then $\rho(\sigma(0), \sigma(t))$ is strictly increasing for $t>0$. Moreover, $\sigma$ does not intersect itself and $\sigma(t) \rightarrow \infty$ as $|t| \rightarrow \infty$. In particular, all nontrivial geodesics seperate $\Re^{2}$ into two connected components.

Proof. Let $\epsilon>0$ be such that $\frac{-\pi}{2}+\epsilon<\int_{S^{-}} \kappa d A \leq \int_{S^{+}} \kappa d A<\frac{\pi}{2}-\epsilon$. Let us fix $t>0$, and let $\mu$ be a geodesic of shortest length joining $\sigma(0)$ to $\sigma(t)$. Consider the interior angle $\theta(\geq 0)$ at $\sigma(t)$ between $\mu$ and $\sigma$. From the Gauss Bonnet theorem it follows that $\theta<\pi / 2-\epsilon$. Let $\delta>0$ be arbitrarily small. Let us consider a point $p$ on $\mu$ close to $\sigma(t)$, and join $p$ to $\sigma(\tau)$ for $\tau \in(t-\delta, t)$ by the unique shortest geodesic. Now, by using the formula for 
the first variation of arclength,

$$
\left.(d / d \tau)\{\rho(p, \sigma(t))-\rho(p, \sigma(\tau))\}\right|_{\tau=t}=-\cos (\theta) .
$$

Therefore, by using the triangle inequality,

$$
\begin{aligned}
\{\rho(\sigma(0), \sigma(t))-\rho(\sigma(0), \sigma(\tau))\} /(t-\tau) & \geq(1 / 2) \sin (\epsilon) ; \\
\tau & \in(t-\delta, t), \text { and } \delta \text { is small enough. }
\end{aligned}
$$

Therefore, it follows that $\rho(\sigma(0), \sigma(t)) \geq(1 / 2) \sin (\epsilon)(t)$ for arbitrary $t>0$. Now, the conclusions of the lemma follows at once.

5.2. Existence and uniqueness of solutions of (3.2). We fix a nontrivial geodesic $\sigma: \Re \rightarrow \Re^{2}$ and parameterize it by arclength $t$. The component of $\Re^{2} \backslash \sigma$ such that $\dot{\sigma}(t)$ and the unit inward normal to the component at $\sigma(t)$ are positively oriented will be called the component above $\sigma$ (denoted by $\Re_{+}$), and the other component is said to be below $\sigma$ (and denoted by $\Re_{-}$). We will specify initial data for $u^{1}, u^{2}$ on $\sigma$ as follows:

$$
u\left(y^{1}, 0\right)=\sigma\left(y^{1}\right) .
$$

$\frac{\partial u}{\partial y^{2}}\left(y^{1}, 0\right)$ is the unit normal to $\sigma$ (with respect to $g$ ) pointing into $\Re_{+}$.

We will prove the existence of a unique $C^{\infty}$ solution in $\Re_{+}$. This proof follows along the ideas of Ladyzhenskaya and Shubov in [LS84].

For $b>0$ let

$$
D_{b}=\left\{\left(y^{1}, y^{2}\right) \in \Re^{2}: 0 \leq y^{2} \leq b-\left|y^{1}\right|\right\} .
$$

The base of $D_{b}$ is $\left\{\left(y^{1}, 0\right) \in \Re^{2}:-b \leq y^{1} \leq b\right\}$ on which Cauchy data has been specified for Servants' equations. We will show that there is a unique smooth solution on $D_{b}$ for all $b>0$. It then follows that there exists a unique smooth solution $\left(y^{1}, y^{2}\right) \mapsto u\left(y^{1}, y^{2}\right): \Re^{2} \rightarrow \Re^{2}$. We will then use assumption (A) to show that this mapping is a diffeomorphism onto $\Re^{2}$, which completes the proof of the main theorem.

In order to carry out this procedure it becomes convenient to work with a metric $g$ such that $u \mapsto \Gamma_{j, \kappa}^{i}(u)$ has bounded partial derivatives. This is done by modifying the metric $g$ outside of a compact set in $\Re^{2}$. Since for a solution $\left(y^{1}, y^{2}\right) \mapsto u\left(y^{1}, y^{2}\right),\left\|\frac{\partial}{\partial x^{i}}\right\|_{g}=1$ and the base of $D_{b}$ is mapped by the geodesic $\sigma$, it follows that $u\left(D_{b}\right)$ is contained in $B_{(1+\sqrt{2}) b}(\sigma(0))$, the ball of radius $(1+\sqrt{2}) b$ around $\sigma(0)$ with respect to the topological metric induced by $g$. Thus, any modification of $g$ outside of $B_{(1+\sqrt{2}) b}(\sigma(0))$ will not affect the solution, $u$, on $D_{b}$. Therefore, without any loss of genarality we assume that the metric coefficients of $g$ with respect to the $u$-coordinate system are constant outside of $B_{(1+\sqrt{2}) b}(\sigma(0))$.

Let $T>(1+\sqrt{2}) b$, and define $u\left(y^{1}, 0\right)=\sigma\left(y^{1}\right)$ for $y^{1} \in[-b, b]$. Extend $u\left(y^{1}, 0\right)$ to $y^{1} \in[-T, T]$ smoothly such that $u(-T, 0)=u(T, 0)$ (in what follows we will see that the choice of the extension does not influence the solution of the Servants' equations on $D_{b}$ ). 
Define $\frac{\partial u}{\partial y^{2}}\left(y^{1}, 0\right)$ for $y^{1} \in[-b, b]$ such that $\frac{\partial}{\partial x^{1}}$ and $\frac{\partial}{\partial x^{2}}$ are unit vectors on $u([-T, T], 0)$, and make angles $\frac{\pi}{4}$ and $\frac{3 \pi}{4}$ with $\dot{\sigma}$ on $\sigma[-b, b]$. Extend $\frac{\partial u}{\partial y^{2}}\left(y^{1}, 0\right)$ smoothly to $[-T, T]$ such that $\frac{\partial u}{\partial y^{2}}(-T, 0)=\frac{\partial u}{\partial y^{2}}(T, 0)$.

Let $a>b$, and let $C_{(T, a)}:=\left\{\left(y^{1}, y^{2}\right) \in \Re^{2}: y^{1} \in[-T, T] ; y^{2} \in[0, a]\right\}$. Consider $C_{(T, a)}$ as the cylinder $S_{T} \times[0, a]$, where $\left.S_{T}:=[-T, T] /\{-T, T\}\right)$.

We will show that Servants' equations admit a smooth solution $u$ on $C_{(T, a)}$ (this means that $u$ is $2 T$-periodic with respect to the $y^{1}$-variable), which satisfies specified boundary conditions.

For positive integers $s$ let $H_{T}^{s}$ denote the Sobolev space on $S_{T}$,

$$
H_{T}^{s}=\left\{v \in L^{2}\left(S_{T}\right): \int_{-T}^{T}\left|\frac{d^{k}}{d \theta^{k}} v(\theta)\right|^{2} d \theta<\infty, k=0, \ldots, s\right\} .
$$

The notation $\|v\|_{s}$ denotes the usual norm

$$
\left(\sum_{k=0}^{s} \int_{-T}^{T}\left|\frac{d^{k}}{d \theta^{k}} v(\theta)\right|^{2} d \theta\right)^{1 / 2}
$$

Let $\left\{\omega_{K}(\theta)\right\}_{k=-\infty}^{\infty}$ denote the orthogonal basis of $H_{T}^{s}$,

$$
\omega_{k}(\theta)= \begin{cases}\frac{1}{2 T}, & k=0, \\ \frac{1}{T} \cos \left(\frac{k \pi \theta}{T}\right), & k<0, \\ \frac{1}{T} \sin \left(\frac{k \pi \theta}{T}\right), & k>0 .\end{cases}
$$

We will find a sequence of functions on $C_{(T, a)}$,

$$
u_{N}^{i}\left(y^{1}, y^{2}\right)=\sum_{k=-N}^{N} a_{k, N}^{i}\left(y^{2}\right) \omega_{k}\left(y^{1}\right), \quad i=1,2, N=1,2, \ldots,
$$

such that for each $N$,

$$
\begin{aligned}
& \int_{-T}^{T}\left\{\left[\frac{\partial^{2}}{\partial\left(y^{2}\right)^{2}}-\frac{\partial^{2}}{\partial\left(y^{1}\right)^{2}}\right]\right. u_{N}^{i}(y)-\sum_{k, m=1}^{2} \Gamma_{k, m}^{i}\left(u_{N}(y)\right) \\
&\left.\times\left[\frac{\partial u_{N}^{k}}{\partial y^{2}} \frac{\partial u_{N}^{m}}{\partial y^{2}}-\frac{\partial u_{N}^{k}}{\partial y^{1}} \frac{\partial u_{N}^{m}}{\partial y^{1}}\right]\right\} \omega^{l}\left(y^{1}\right) d y^{1}=0, \\
& l=-N, \ldots, N \cdot, y^{2} \in[0, a] .
\end{aligned}
$$

Equation (5.2) yields a system of $(4 N+2)$ second-order ordinary differential equations for $\left\{a_{k, N}^{i}\left(y^{2}\right)\right\}_{k \in\{-N, \ldots, N\}}, i=1,2$ (Note that the nonlinear term $\int_{-T}^{T} \Gamma_{k, m}^{i}\left(u_{N}(y)\right) \omega^{k}\left(y^{1}\right) d y^{1}$ is to be considered as a function of $a_{k, N}^{i}$ which makes the system of equations nonlinear and coupled with each other.)

We specify the boundary conditions

$$
\begin{aligned}
a_{k, N}^{i}(0) & =\int_{-T}^{T} u^{i}\left(y^{1}, 0\right) \omega_{k}\left(y^{1}\right) d y^{1}, \\
\left(d / d y^{2}\right)\left(a_{k, N}^{i}\right)(0) & =\int_{-T}^{T} \frac{\partial u^{i}}{\partial y^{2}}\left(y^{1}, 0\right) \omega_{k}\left(y^{1}\right) d y^{1} .
\end{aligned}
$$


We will show that $\left\{u_{N}^{i}\left(y^{1}, y^{2}\right)\right\}$ contains a subsequence which converges to a smooth function $\left\{u_{\infty}^{i}\left(y^{1}, y^{2}\right)\right\}$ on $C_{(T, a)}$, and that $u_{\infty}$ satisfies Servants' equations on $C_{(T, a)}$, for the modified metric.

For convenience, replace $u_{N}^{i}$ by $u^{i}, u_{N}$ by $u, y^{1}$ by $\theta$, and $y^{2}$ by $t$. Let

$$
\Im^{i}(u):=u^{i}+\sum_{l, m=1}^{2} \Gamma_{\ell, m}^{i}(u)\left(\frac{\partial u^{\ell}}{\partial t} \frac{\partial u^{m}}{\partial t}-\frac{\partial u^{\ell}}{\partial \theta} \frac{\partial u^{m}}{\partial \theta}\right), \quad i=1,2,
$$

and $\Im(u):=\left(\Im^{1}(u), \Im^{2}(u)\right)$. Then $(5.2)$ can be rewritten as

$$
\int_{-T}^{T}\left[\left(\oplus u^{i}\right)-\Im^{i}(u)\right] \omega^{k}(\theta) d \theta=0,
$$

where $\oplus=\left[\left(\frac{\partial^{2}}{\partial t^{2}}-\frac{\partial^{2}}{\partial \theta^{2}}\right)+1\right]$. Using (5.1), (5.2), and (5.5) we may write

$$
\begin{aligned}
& \int_{-T}^{T}\left\{\left(\oplus u^{i}\right) u_{t}^{i}+\left(\oplus u_{t}^{i}\right) u_{t t}^{i}+\left(\oplus u_{\theta}^{i}\right) u_{\theta t}^{i}\right. \\
& \left.+\left(\oplus u_{t t}^{i}\right) u_{t t t}^{i}+\left(\oplus u_{t \theta}^{i}\right) u_{t t \theta}^{i}+\left(\oplus u_{\theta \theta}^{i}\right) u_{\theta \theta t}^{i}\right\} d \theta \\
& =\int_{-T}^{T}\left\{\Im^{i}(u) u_{t}^{i}+\left(\frac{\partial}{\partial t} \Im^{i}(u)\right) u_{t t}^{i}+\left(\frac{\partial}{\partial \theta} \Im^{i}(u)\right) u_{\theta t}^{i}\right. \\
& \left.+\left(\frac{\partial^{2}}{\partial t^{2}} \Im^{i}(u)\right) u_{t t t}^{i}+\left(\frac{\partial}{\partial \theta^{2}} \Im^{i}(u)\right) u_{\theta \theta t}^{i}+\left(\frac{\partial^{2}}{\partial \theta \partial t} \Im^{i}(u)\right) u_{\theta t t}^{i}\right\} d \theta, \\
& \quad i=1,2 .
\end{aligned}
$$

Let

$$
\begin{aligned}
Z(t):=\sum_{i=1}^{2} \int_{-T}^{T}\left\{\left(u^{i}\right)^{2}+2\left(u_{t}^{i}\right)^{2}+2\left(u_{\theta t}^{i}\right)^{2} 2\left(u_{\theta}^{i}\right)^{2}\right. \\
+2\left(u_{t t}^{i}\right)^{2}+2\left(u_{\theta \theta}^{i}\right)^{2}+\left(u_{t t t}^{i}\right)^{2}+\left(u_{\theta \theta \theta}^{i}\right)^{2} \\
\left.+3\left(u_{\theta t}^{i}\right)^{2}+2\left(u_{t \theta \theta}^{i}\right)^{2}+2\left(u_{t t \theta}^{i}\right)^{2}\right\} d \theta .
\end{aligned}
$$

By repeated application of the integration by parts formula $\int_{T}^{T} v_{\theta} w d \theta=$ $-\int_{T}^{T} v w_{\theta} d \theta$, for and $C^{1}$ functions $v, w$ defined on the circle, we could simplify each term in the left-hand side of (5.6) to look like $\int_{T}^{T} v v_{t} d \theta$. Now we observe that the sum of the left-hand sides of (5.6) for $i=1,2$ is

$$
\frac{1}{2} \frac{d}{d t} Z(t)
$$

Since $\left\{\Gamma_{j, k}^{i}\right\}$ are constants outside of a compact set, it follows that the righthand side of $(5.6)$ is bounded by $c\left(Z(t)+Z^{3}(t)\right)$ for some $c>0$, which depends only on the bounds on the partial derivatives of $\left\{\Gamma_{j, k}^{i}(u)\right\}$ with respect to $u^{1}$ and $u^{2}$, and hence independent of $N$. Therefore we have

$$
\frac{d}{d t} Z(t) \leq c\left\{Z(t)+Z^{3}(t)\right\}
$$


Since $Z(0) \leq \lambda$, for some $\lambda$ which is independent of $N$ also, it follows that there exists $\tau>0$ such that $Z(t)$ exists for $t \in[0, \tau]$ and remains bounded. Now from the Sobolev embedding lemma, it follows that a subsequence of $\left\{u_{N}\right\}$ converges to some $u_{\infty} \in C^{2}\left(C_{(T, \tau)}\right)$. Since $(\oplus-\Im): C^{2}\left(C_{(T, \tau)}\right) \rightarrow C^{\circ}\left(C_{(T, \tau)}\right)$ is continuous, it follows that $u_{\infty}$ satisfies Servants' equations on $C_{(T, \tau)}$.

Since the solution $u$ is such that $\left\|\frac{\partial}{\partial x_{i}}\right\|=1$, and since the metric coefficients $g_{i j}(u)$ are bounded on $\Re^{2}$, it follows that there exists an a priori constant $\mu>0$ such that $\left\|\frac{\partial u_{\infty}}{\partial y^{i}}\right\|<\mu, i=1,2$, on $C_{(T, t)}$, and $\mu$ does not depend on $t$. From the equation (3.1) it follows that (after possibly redefining $\mu$ to be a larger constant) $\left|\frac{\partial^{2} u_{\infty}}{\partial x_{1} x_{2}}\right|<\mu$ on $C_{(T, t)}$ (We have used the fact that $g_{i, j}$ are constant outside of a compact set here.) Also, the same conclusion holds for $u_{n}$ for large enough $n$.

We claim that there exists a continuous function $\eta:[0, \infty) \rightarrow \Re$ (independant of $\tau)$ such that $\left|\frac{\partial^{2} u^{j}}{\partial y^{i 2}}\right|<\eta\left(y^{2}\right)$ on $C_{(T, \tau)}$. Let us define $X_{i}=\partial / \partial x^{i}$, $i=1,2$, where $\left(x^{1}, x^{2}\right)=(1 / 2)\left(y^{1}+y^{2}, y^{1}-y^{2}\right)$ (as defined in section 3). Since $\nabla_{X_{1}} X_{2}=0$ and $\left[X_{1}, X_{2}\right]=0$, it follows that

$$
\nabla_{X_{1}} \nabla_{X_{2}} X_{2}=R\left(X_{1}, X_{2}\right) X_{2},
$$

where $R$ is the curvature tensor of the Riemannian metric $g$. Since $g_{i, j}$ are constant outside of a compact set, it follows that there exists a constant $c>0$ such that $|\langle R(p, q) r, s\rangle| \leq c$ for all unit tanget vectors $p, q, r, s$ at an arbitrary point in $\Re^{2}$. Therefore,

$$
\begin{aligned}
\left|L_{X_{1}}\left\|\nabla_{X_{2}} X_{2}\right\|_{g}^{2}\right| & =2\left|\left\langle\nabla_{X_{2}} X_{2}, R\left(X_{1}, X_{2}\right) X_{2}\right\rangle\right| \\
& \leq 2 c\left\|\nabla_{X_{2}} X_{2}\right\|_{g} .
\end{aligned}
$$

Therefore, we have

$$
\left\|\nabla_{X_{2}} X_{2}\left(y^{1}, y^{2}\right)\right\|_{g} \leq 4 c\left(y^{2}\right)+d
$$

where $d=\max \left\{\left\|\nabla_{X_{2}} X_{2}\left(y^{1}, 0\right)\right\|_{g} \mid y^{1} \in[-b, b]\right\}$.

Let us now write $\nabla_{X_{2}} X_{2}$ in terms of local coordinate expressions:

$$
\nabla_{X_{2}} X_{2}=\sum_{i=1,2} \frac{\partial^{2} u^{i}}{\partial\left(x^{2}\right)^{2}} \frac{\partial}{\partial u^{i}}+\sum_{i, j, k=1}^{2} \frac{\partial u^{i}}{\partial x^{2}} \frac{\partial u^{j}}{\partial x^{2}} \Gamma_{i j}^{k} \frac{\partial}{\partial u^{k}} .
$$

It now follows that there exists a constant $d_{1}>0$ such that

$$
\left\|\sum_{i=1}^{2} \frac{\partial^{2} u^{i}}{\partial\left(x^{2}\right)^{2}} \frac{\partial}{\partial u^{i}}\right\|_{g} \leq 4 c t+d+d_{1},
$$

and the constants $c, d, d_{1}$ are independent of $\tau$. A similar bound holds for $\left\|\sum_{i=1}^{2} \frac{\partial^{2} u^{i}}{\partial\left(x^{1}\right)^{2}} \frac{\partial}{\partial u^{i}}\right\|_{g}$. Since $g_{i, j}$ are constant outside of a compact set, the claim follows immediately.

Since the right-hand side of (5.6) depends linearly on third order derivatives, and since we have established bounds for first and second derivatives, it follows that there exists a continuous function $\beta:[0, \infty) \rightarrow \Re$, such that for large 
enough $N, u_{N}$ satisfies

$$
\frac{d}{d t} Z(t) \leq \beta(t)(Z(t)+1)
$$

Hence it follows that the domain of definition of a $C^{2}$ solution is arbitrary: i.e., $u_{\infty} \in C^{2}\left(C_{(T, t)}\right) \forall t>0$.

In particular, we have found a $C^{2}$ solution to Servants' equations on $C_{(T, b)}$. Since the method we used to obtain bounds is applicable for higher order derivatives also, by using a diagonal process if necessary, we obtain a $C^{\infty}$ solution to Servants' equations, $u \in C^{\infty}\left(C_{(T, \infty)}\right)$.

Now we have completed the proof of the existence of a $C^{\infty}$-solution of (3.2) on $D_{b}$ satisfying the specified boundary conditions.

We will now show that this solution is unique. If $\left\{u^{i}\right\}_{i=1,2}$ and $\left\{\bar{u}^{i}\right\}_{i=1,2}$ are two solutions on $D_{b}$, then the difference $\mu^{i}=u^{i}-\bar{u}^{i}$ satisfies the linear equation

$$
\left(\frac{\partial^{2}}{\partial\left(y^{1}\right)^{2}}-\frac{\partial^{2}}{\partial\left(y^{2}\right)^{2}}\right) \mu^{i}=d_{j}^{i}(y) \frac{\partial \mu^{i}}{\partial y^{j}}+\Psi^{i}(y) \mu^{i}
$$

where $\mu^{i}\left(0, y^{2}\right)=0$ and $\frac{\partial^{\mu^{i}}}{\partial y^{1}}\left(0, y^{2}\right)=0$ where $d_{j}^{i}$ and $\Psi^{i}$ are $C^{\infty}$ functions on $D_{b}$. Then it follows easily that $\mu^{i} \equiv 0$ on $D_{b}$. Thus $u^{i}$ is unique on $D_{b}$. Hence the solutions on $\left\{D_{b}\right\}_{0<b<\infty}$ piece together to give a $C^{\infty}$-solution of (3.2) on $\Re_{+}$(and similarly on $\Re^{2}$ ) for the original metric subject to specified boundary conditions.

5.3. Existence of Tchebychev nets. Now let $D_{b}$ be a triangle such that $u: D_{b} \rightarrow$ $\Re^{2}$ is a diffeomorphism into $\Re^{2}$.

$$
\begin{aligned}
\text { Let } \Delta_{b} \text { denote } u\left(D_{b}\right) \text { and } \\
\mu_{1}=\sigma([-b, b]), \\
\mu_{2}=u(\{(b-\theta, \theta): 0 \leq \theta \leq b\}), \\
\mu_{3}=u(\{(-b+\theta, \theta): 0 \leq \theta \leq b\}), \\
\rho_{1}=u(0, b), \\
\rho_{2}=u(-b, 0), \\
\rho_{3}=u(b, 0) .
\end{aligned}
$$

Now $\frac{\partial}{\partial x^{1}}$ is parallel along $\mu_{1} \cup \mu_{2}$ and $\frac{\partial}{\partial x^{2}}$ is parallel along $\mu_{1} \cup \mu_{3}$. Let $\gamma=$ angle between $\frac{\partial}{\partial x^{2}}$ and $\frac{\partial}{\partial x^{1}}$ at $\rho_{1}$.

Then it follows that

$$
\gamma=\int_{\Delta_{b}} \kappa d A+\frac{\pi}{2}
$$

By assumption (A), $\exists \epsilon>0 \ni \int_{V} \kappa d A \in\left(\frac{-\pi}{2}+\epsilon, \frac{\pi}{2}-\epsilon\right)$ for all measurable sets $V$. Hence we conclude that $\gamma \in(\epsilon, \pi-\epsilon)$. Clearly, this conclusion holds for the angle between $\partial / \partial x^{1}$ and $\partial / \partial x^{2}$ at all points in $\Delta_{b}$.

Now suppose that $u: \Re^{2} \rightarrow \Re^{2}$ is not a diffeomorphism. Then, increase $b$ until $\left.u\right|_{D_{b}}$ is not a diffeomorphism. Let $b_{\circ}$ be the smallest such value. Then there exist $p_{2}, p_{3} \in \partial D_{b}$ such that $u\left(p_{2}\right)=u\left(p_{3}\right)$. Let $q_{i}=u\left(p_{i}\right), i=1,2$. 
Without loss of generality we may assume that $q_{3} \in \mu_{3} \backslash\left\{\rho_{1}, \rho_{2}\right\}$. Let $\bar{\theta}$ and $\hat{\theta}$ be the angle between $\frac{\partial}{\partial x^{2}}$ and $\frac{\partial}{\partial x^{1}}$ at $q_{2}$ and $q_{3}$, respectively.

In what follows, by a fiber triangle we mean the image under $u$ of a triangle in the $y$-plane with its base on the $y_{1}$-axis, and its sides with slopes +1 and -1 .

Case 1: $q_{2} \in \mu_{3}$. Let $R_{1}$ be the region enclosed by the segment of $\mu_{3}$ which lies betwen $q_{3}$ and $q_{2}$. By computing holonomy along $\partial \hat{\Delta}$ we conclude that

$$
\pi-\hat{\theta}+\bar{\theta}=\int_{\hat{R}_{1}} \kappa d A \in(-\pi / 2-\epsilon, \pi / 2-\epsilon) .
$$

Let $R_{2}$ and $R_{3}$ be the fiber triangles of which two vertices are $\rho_{2}, q_{3}$ and $\rho_{2}, q_{2}$ respectively. Then

$$
\hat{\theta}=\int_{R_{2}} \kappa d A+\frac{\pi}{2}, \quad \bar{\theta}=\int_{R_{3}} \kappa d A+\frac{\pi}{2} .
$$

Therefore,

$$
\int_{R_{3}-R_{2}} \kappa d A=-\pi+\int_{\hat{R}_{1}} \kappa d A<-\pi / 2-\epsilon
$$

which contradicts our assumption on the bounds on the integral of the Gauss curvature over arbitrary subsets.
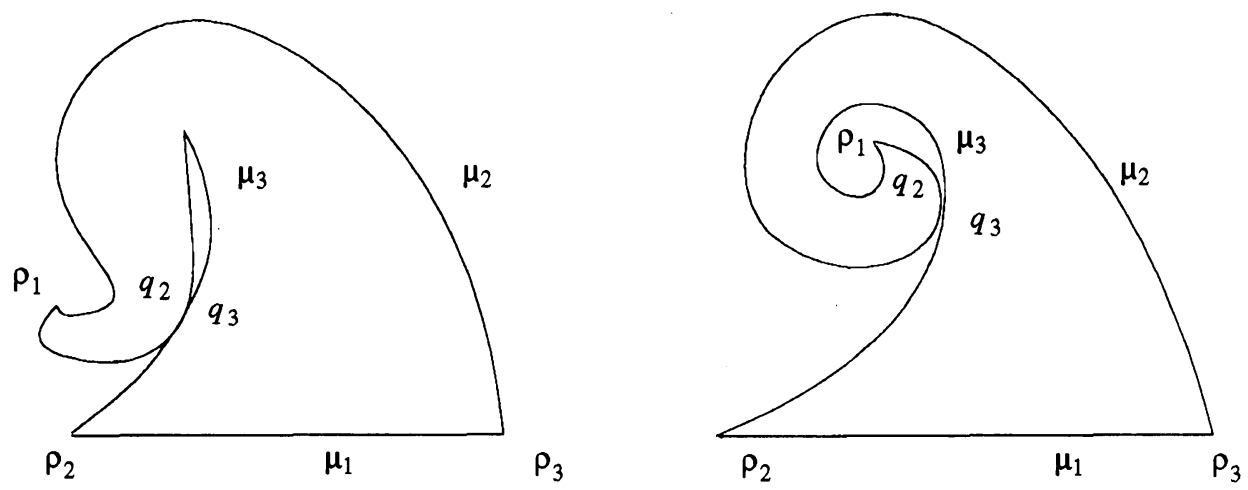

Case 2: $q_{2} \in \mu_{2}$. Let $R_{1}$ be the region enclosed by the segment of $\mu_{3}$ which lies between $q_{3}$ and $\rho_{1}$, and the segment of $\mu_{2}$ which lies between $\rho_{1}$ and $q_{2}$. By computing holonomy along $\partial \hat{\Delta}$ we conclude that

$$
2 \pi+\gamma-\hat{\theta}-\bar{\theta}=\int_{\hat{\Delta}} \kappa d A \in(-\pi / 2+\epsilon, \pi / 2-\epsilon) .
$$

Define $R_{2}$ and $R_{3}$ as in Case 1 , and by an argument similar to Case 1 we observe that

$$
\int_{R_{3}+R_{2}} \kappa d A>\pi+2 \epsilon .
$$

This is a contradiction. 
Thus, we conclude that $u:[0, \infty) \times \Re \rightarrow \Re^{2}$ is a diffeomorphism into $\Re^{2}$. A similar construction for the lower half plane allows us to extend $u$ to $u: \Re^{2} \rightarrow \Re^{2}$, a diffeomorphism into $\Re^{2}$.

We now prove that $u$ is onto. Let $W=u\left(\Re^{2}\right)$. First note that from the above that the counterclockwise angle between $\frac{\partial}{\partial x^{1}}$ and $\frac{\partial}{\partial x^{2}}$ (call it $\left.\gamma\right)$ is in $(\epsilon, \pi-\epsilon)$. Let $\hat{g}$ denote the Riemannian metric $\left(d x^{1}\right)^{2}+2 \cos \gamma d x^{1} d x^{2}+\left(d x^{2}\right)^{2}$.

Then $u: \Re^{2} \rightarrow W$ is an isometry, when $\Re^{2}$ is endowed with $\hat{g}$ and $W$ is endowed with $g$.

In order to show that $W=\Re^{2}$, it suffices to show that if $\lambda:[0, \infty] \rightarrow \Re^{2}$ is a smooth curve of infinite euclidean length, then it has infinite length with respect to $\hat{g}$ as well. But this follows since

$$
\begin{aligned}
\|\dot{\lambda}(t)\| \hat{g} & =\sqrt{\left(\frac{\partial \lambda_{1}}{\partial t}\right)^{2}+\left(\frac{\partial \lambda_{2}}{\partial t}\right)^{2}+2 \cos \gamma \frac{d \lambda_{1}}{d t} \frac{d \lambda_{2}}{d t}} \\
& \geq\|\dot{\lambda}(t)\|_{2} \sqrt{(1-|\cos \gamma|)} \\
& \geq\|\dot{\lambda}(t)\|_{2} \sqrt{(1-\sin (\epsilon))}
\end{aligned}
$$

where $\|\dot{\lambda}(t)\|_{2}$ denotes the Euclidean length of $\dot{\lambda}(t)$. This concludes the proof of the main theorem.

\section{REFERENCES}

[Haz80] J. N. Hazzidakis, Ueberienge Iegenschaften der Flachen Mit Constatem Krummungsmaass, J. Reine Angew. Math. (Crelle) 88 (1880), 68-73.

[LS84] O. A. Ladyzhenskaya and V. Shubov, Unique solvability of the Cauchy problem of equations of the two dimensional chiral fields with values in a Riemannian manifold, J. Soviet Math. 25 (1984), 855-864,.

[Pip81] A. C. Pipkin, Plane traction problem for inextensible networks, Quart. J. Mech. Appl. Math. 34 (1984), 415-429.

[Riv59] R. S. Rivlin, The deformation of a membrane formed by inextensible cords, Arch. Rational Mech. Anal. 2 (1959), 447-476.

[Sam90] S. L. Samelson, Tchebychev nets on two dimensional Riemannian manifolds, Ph.D. Thesis, Carnagie Mellon University, Pittburgh, 1990.

[Sam91a] _ _ Tchebychev nets on spheres, Quart. Appl. Math. 69 (1991), 11-18.

[Sam91b] , Global Tchebychev nets on complete Riemannian manifolds, Arch. Rational Mech. Anal. 114 (1991), 237-254.

[Tch78] P. L. Tchebychev, Sur l'coupe des vetements, Assoc. France pour L'Advancement des Sci., Congres de Paris, 1878, pp. 154-155.

Department of Mathematics and Computer Science, SUNY College at New Paltz, New Paltz, New York 12561

E-mail address: samelsos@matrix.newpaltz.edu

Department of Electrical Engineering and the Systems Research Center, Universtiy of Maryland,College Park, Maryland 20742

E-mail address: wpd@eng. umd.edu 